EUROPEAN UNIVERSITY INSTITUTE

DEPARTMENT OF ECONOMICS

EUI Working Paper ECO No. 2001/1

The Asymptotic Variance of the

Estimated Roots in a Cointegrated

Vector Autoregressive Model

SøREn JOHANSEN

BADIA FIESOLANA, SAN DOMENICO (FI) 


\begin{abstract}
All rights reserved.
No part of this paper may be reproduced in any form without permission of the author.
\end{abstract}

\author{
(C)2001 Søren Johansen \\ Printed in Italy in February 2001 \\ European University Institute \\ Badia Fiesolana \\ I-50016 San Domenico (FI) \\ Italy
}




\title{
The Asymptotic Variance of the Estimated Roots in a Cointegrated Vector Autoregressive Model
}

\author{
Søren Johansen \\ Department of Economics \\ European University Institute \\ Badia Fiesolana \\ 50016 San Domenico di Fiesole (FI) \\ Italy \\ http://www.iue.it/Personal/Johansen/ \\ January 17, 2001
}

\begin{abstract}
We show that the asymptotic distribution of the estimated stationary roots in a vector autoregressive model is Gaussian. A simple expression for the asymptotic variance in terms of the roots and the eigenvectors of the companion matrix is derived. The results are extended to the cointegrated vector autoregressive model and we discuss the implementation of the results for complex roots.
\end{abstract}

Key words: Autoregressive process; estimated eigenvalues; error correction model; asymptotic distribution

JEL classification: C32 


\section{Introduction}

In a stationary vector autoregressive model the estimated coefficients are asymptotically Gaussian, see Anderson (1971). In this note we show that the asymptotic distribution of the estimated stationary simple roots is also Gaussian, and derive a simple expression for the asymptotic variance matrix. We thereby extend and simplify results by Wymer (1972).

In order to prove this, we give a necessary and sufficient condition for a root of the characteristic polynomial to be a simple root, that is have multiplicity one, and use that a simple root is a continuous and differentiable function of the coefficients in the model.

The results are shown to hold also for the stationary roots of a cointegrated vector autoregressive model by investigating the companion form for the stationary cointegrating relations and the differences. Finally it is shown how the Arcsin transformation helps eliminate a singularity of the variance when a root is close to one.

In Section 2 the roots and the companion form are investigated, and in Section 3 we find the asymptotic distribution of the simple eigenvalues. In Section 4 we extend the result to the cointegrated vector autoregressive model and discuss some issues in relation to the calculation of the variances. We conclude in Section 5 with a few simulations to illustrate the results.

\section{The roots of an autoregressive process}

Let the $n$-dimensional process $X_{t}$ be given by the vector autoregressive model

$$
X_{t}=\Pi_{1} X_{t-1}+\Pi_{2} X_{t-2}+\Pi_{3} X_{t-3}+\varepsilon_{t},
$$

where $\varepsilon_{t}$ are i.i.d. with mean zero and variance matrix $\Omega$, and the initial conditions are fixed. We have taken three lags for notational convenience. We define the characteristic polynomial as

$$
\Pi(\rho)=\rho^{3} I_{n}-\rho^{2} \Pi_{1}-\rho \Pi_{2}-\Pi_{3},
$$


with determinant $|\Pi(\rho)|$ and derivative

$$
\dot{\Pi}(\rho)=3 \rho^{2} I_{n}-2 \rho \Pi_{1}-\Pi_{2} .
$$

We let $\rho_{1}, \ldots, \rho_{m}$ denote the roots of

$$
|\Pi(\rho)|=0,
$$

and associate with each root $\rho$ the $n$-vectors $\tau_{\rho}$ and $\kappa_{\rho}$, with the property that

$$
\tau_{\rho}^{*} \Pi(\rho)=0, \Pi(\rho) \kappa_{\rho}=0 .
$$

Note that in general the roots and the vectors $\tau_{\rho}$ and $\kappa_{\rho}$ may be complex valued. Hence if $\tau=\tau_{r}+i \tau_{i}$, we use the notation $\tau^{*}=\tau_{r}^{\prime}-i \tau_{i}^{\prime}=$ $\bar{\tau}_{\rho}^{\prime}$. The vector $\bar{\tau}_{\rho}$ corresponds to the root $\bar{\rho}$. Note also that if $\rho$ is not a simple root, the vectors $\tau_{\rho}$ and $\kappa_{\rho}$ are not unique. For any full rank (complex) matrix $a(n \times m), m<n$, we define $a_{\perp}(n \times(n-m))$ of full rank, so that $a^{*} a_{\perp}=0$. We give in the next theorem a condition on $\Pi(\rho)$ to ensure that $\rho_{0}$ is a simple root. The result is a slight reformulation of Theorem 3 from Johansen and Schaumburg (1998).

Theorem 1 If $\rho_{0}$ is a root of the equation

$$
|\Pi(\rho)|=0,
$$

then $\Pi\left(\rho_{0}\right)=a b^{*}$ where $a$ and $b$ are $n \times r$ matrices of full rank for some $r<n$. It then holds that $\rho_{0}$ is an $(n-r)-$ double root if and only if

$$
\left|a_{\perp}^{*} \dot{\Pi}\left(\rho_{0}\right) b_{\perp}\right| \neq 0 .
$$

In this case

$$
|\Pi(\rho)|=\left(\rho-\rho_{0}\right)^{n-r} \frac{\left|a^{*} a\right|\left|b^{*} b\right|}{\left|\left(a, a_{\perp}\right)^{*}\right|\left|\left(b, b_{\perp}\right)\right|}\left|a_{\perp}^{*} \dot{\Pi}\left(\rho_{0}\right) b_{\perp}\right|\left(1+O\left(\rho-\rho_{0}\right)\right),
$$

and $\Pi(\rho)^{-1}$ has a pole of order 1 at $\rho_{0}$ :

$$
\Pi(\rho)^{-1}=\frac{1}{\rho-\rho_{0}} b_{\perp}\left(a_{\perp}^{*} \dot{\Pi}\left(\rho_{0}\right) b_{\perp}\right)^{-1} a_{\perp}^{*}+O(1) .
$$


Proof. The matrix polynomial $\Pi(\rho)$ has the expansion at $\rho=\rho_{0}$

$$
\Pi(\rho)=\Pi\left(\rho_{0}\right)+\left(\rho-\rho_{0}\right) \tilde{\Pi}(\rho),
$$

where the polynomial $\tilde{\Pi}(\rho)$ satisfies $\tilde{\Pi}\left(\rho_{0}\right)=\dot{\Pi}\left(\rho_{0}\right)$. Because $\Pi\left(\rho_{0}\right)$ is singular, it has the representation

$$
\Pi\left(\rho_{0}\right)=a b^{*},
$$

where $a$ and $b$ are $n \times r$ matrices of rank $r<n$. Now multiply (8) by the full rank matrices $\left(a, a_{\perp}\right)^{*}$ and $\left(b, b_{\perp}\right)$ and we find that

$$
\begin{aligned}
& \left(a, a_{\perp}\right)^{*} \Pi(\rho)\left(b, b_{\perp}\right) \\
& =\left[\left(\begin{array}{cc}
a^{*} a b^{*} b & a^{*} \dot{\Pi}\left(\rho_{0}\right) b_{\perp} \\
0 & a_{\perp}^{*} \dot{\Pi}\left(\rho_{0}\right) b_{\perp}
\end{array}\right)+O\left(\rho-\rho_{0}\right)\right]\left(\begin{array}{cc}
I_{r} & 0 \\
0 & \left(\rho-\rho_{0}\right) I_{n-r}
\end{array}\right) .
\end{aligned}
$$

This relation immediately implies the result about the determinant (6) and the expression for

$$
\lim _{\rho \rightarrow \rho_{0}}\left(\rho-\rho_{0}\right) \Pi(\rho)^{-1}=\lim _{\rho \rightarrow \rho_{0}}\left(\rho-\rho_{0}\right)\left(b, b_{\perp}\right)\left[\left(a, a_{\perp}\right)^{*} \Pi(\rho)\left(b, b_{\perp}\right)\right]^{-1}\left(a, a_{\perp}\right)^{*},
$$

which shows (7).

As a special case of Theorem 1 we find that $\rho$ is a simple root $(r=1)$ if and only $\Pi(\rho)=a b^{*}$, with $a$ and $b$ of rank $n-1$, and $a_{\perp}^{*} \dot{\Pi}(\rho) b_{\perp} \neq 0$. In this case we associate to $\rho$ two vectors $\tau_{\rho}=a_{\perp}$ and $\kappa_{\rho}=b_{\perp}$, which we normalize by

$$
\tau_{\rho}^{*} \dot{\Pi}(\rho) \kappa_{\rho}=1
$$

see $(5)$.

We give a result on the differentiablity of a simple eigenvalue as function of the coefficient matrices, see Magnus and Neudecker (1995, p. 161)

Theorem 2 Let $\Pi_{0}(\cdot)$ be a matrix polynomial with a simple root at $\rho_{0}$. If $\Pi(\cdot)$ is close to $\Pi_{0}(\cdot)$, then it has a simple root close to $\rho_{0}$. This defines a continuous function $\rho(\Pi)$ in a neighborhood of $\Pi_{0}$. The function is also differentiable with differential

$$
d \rho=-\tau_{\rho}^{*}(d \Pi)(\rho) \kappa_{\rho}=-\tau_{\rho}^{*}\left(\rho^{2}\left(d \Pi_{1}\right)+\rho\left(d \Pi_{2}\right)+\left(d \Pi_{3}\right)\right) \kappa_{\rho} .
$$


Proof. From the relations (2) and (3) we find, by taking differentials, that

$$
d\left(\Pi(\rho) \kappa_{\rho}\right)=\dot{\Pi}(\rho) \kappa_{\rho}(d \rho)+(d \Pi)(\rho) \kappa_{\rho}+\Pi(\rho)\left(d \kappa_{\rho}\right)=0 .
$$

Multiplying by $\tau_{\rho}^{*}$ we get, since $\tau_{\rho}^{*} \dot{\Pi}(\rho) \kappa_{\rho}=1$ and $\tau_{\rho}^{*} \Pi(\rho)=0$, that

$$
\tau_{\rho}^{*} \dot{\Pi}(\rho) \kappa_{\rho}(d \rho)+\tau_{\rho}^{*}(d \Pi)(\rho) \kappa_{\rho}+\tau_{\rho}^{*} \Pi(\rho)\left(d \kappa_{\rho}\right)=d \rho+\tau_{\rho}^{*}(d \Pi)(\rho) \kappa_{\rho}=0 .
$$

\subsection{The companion form of the vector autoregres- sive model}

The companion form of $(1)$ is

$$
\tilde{X}_{t}=A \tilde{X}_{t-1}+B \varepsilon_{t}
$$

where

$$
\tilde{X}_{t}=\left(\begin{array}{c}
X_{t} \\
X_{t-1} \\
X_{t-2}
\end{array}\right), A=\left(\begin{array}{ccc}
\Pi_{1} & \Pi_{2} & \Pi_{3} \\
I_{n} & 0 & 0 \\
0 & I_{n} & 0
\end{array}\right), B=\left(\begin{array}{c}
I_{n} \\
0 \\
0
\end{array}\right)
$$

It is well-known that

$$
\left|\rho I_{3 n}-A\right|=|\Pi(\rho)|,
$$

see for instance Johansen (1996, p. 16), which implies that the roots of $|\Pi(\rho)|=0$ are the same as the eigenvalues of $A$. A right eigenvector is

$$
\tilde{\kappa}_{\rho}^{\prime}=\kappa_{\rho}^{\prime}\left(\rho^{2} I_{n}, \rho I_{n}, I_{n}\right),
$$

where $\kappa_{\rho}$ satisfies $\Pi(\rho) \kappa_{\rho}=0$. Similarly a left eigenvector $\tilde{\tau}_{\rho}$ for $A$ satisfies

$$
\tilde{\tau}_{\rho}^{*}=\tau_{\rho}^{*}\left(I_{n}, \rho I_{n}-\Pi_{1}, \rho^{2} I_{n}-\rho \Pi_{1}-\Pi_{2}\right),
$$

where $\tau_{\rho}$ satisfies $\tau_{\rho}^{*} \Pi(\rho)=0$, see (4). Further we find from (12) and (13) that

$$
\tilde{\tau}_{\rho}^{*} \tilde{\kappa}_{\rho}=\tau_{\rho}^{*}\left(3 \rho^{2}-2 \rho \Pi_{1}-\Pi_{2}\right) \kappa_{\rho}=\tau_{\rho}^{*} \dot{\Pi}(\rho) \kappa_{\rho}=1,
$$


by the normalization of $\kappa_{\rho}$ and $\tau_{\rho}$, see (9) and (3). From the equation

$$
A \tilde{\kappa}_{\rho}=\rho \tilde{\kappa}_{\rho},
$$

which is equivalent to $\Pi(\rho) \kappa_{\rho}=0$, we find the differential (10) can be written

$$
d \rho=\tilde{\tau}_{\rho}^{*}(d A) \tilde{\kappa}_{\rho}
$$

In the following we sometimes work with the assumption that all roots are simple roots. In this case they are denoted $\rho_{k}, k=1, \ldots, m$, and the corresponding vectors are $\tau_{k}, \tilde{\tau}_{k}, \kappa_{k}, \tilde{\kappa}_{k}$. From the relation

$$
\tilde{\tau}_{k}^{*} A \tilde{\kappa}_{l}=\rho_{k} \tilde{\tau}_{k}^{*} \tilde{\kappa}_{l}=\tilde{\tau}_{k}^{*} \tilde{\kappa}_{l} \rho_{l},
$$

it follows that for $\rho_{k} \neq \rho_{l}$, the vectors $\tilde{\kappa}_{k}$ and $\tilde{\tau}_{l}$ are orthogonal in the sense that $\tilde{\tau}_{l}^{*} \tilde{\kappa}_{k}=0$.

The relations (14) and (16) can be summarized as

$$
\left(\tilde{\tau}_{1}, \ldots, \tilde{\tau}_{m}\right)^{*}=\left(\tilde{\kappa}_{1}, \ldots, \tilde{\kappa}_{m}\right)^{-1} .
$$

\section{$3 \quad$ Asymptotic distributions}

We first discuss briefly the asymptotic distribution of the estimated coefficients in the autoregresive model, Anderson (1971), and then apply the differentiability of the roots to derive the asymptotic distribution of the estimated roots. The parameters in (1) are estimated by regression and for stationary processes $\left(\left|\rho_{k}\right|<1, k=1, \ldots, m\right)$ it holds that

$$
T^{\frac{1}{2}}\left[\left(\hat{\Pi}_{1}, \hat{\Pi}_{2}, \hat{\Pi}_{3}\right)-\left(\Pi_{1}, \Pi_{2}, \Pi_{3}\right)\right] \stackrel{w}{\rightarrow} N_{n \times 3 n}\left(0, \Omega \otimes \Sigma^{-1}\right) .
$$

The variance matrix $\Sigma$ is defined as

$$
\Sigma=\operatorname{Var}\left(\tilde{X}_{t}\right)=\operatorname{Var}\left(X_{t}^{\prime}, X_{t-1}^{\prime}, X_{t-2}^{\prime}\right)^{\prime},
$$

and can be found from

$$
\Sigma=A \Sigma A^{\prime}+B \Omega B^{\prime}
$$


The asymptotic distribution of

$$
T^{\frac{1}{2}}(\hat{A}-A)=B\left(\hat{\Pi}_{1}-\Pi_{1}, \hat{\Pi}_{2}-\Pi_{2}, \hat{\Pi}_{3}-\Pi_{3}\right)
$$

is therefore Gaussian in $(3 n \times 3 n)$ dimensions with mean zero and asymptotic variance matrix

$$
\operatorname{as} V \operatorname{ar}\left(T^{\frac{1}{2}}(\hat{A}-A)\right)=B \Omega B^{\prime} \otimes \Sigma^{-1} .
$$

An estimate of the variance $\Omega$ is found from the regression, and $\Sigma$ can be estimated from (17).

Theorem 3 Let $X_{t}$ be the stationary autoregressive process given by (1) with characteristic polynomial (2). If $\rho$ is a simple root of $|\Pi(\rho)|=0$ and the vectors $\tau_{\rho}$ and $\kappa_{\rho}$ satisfy $\tau_{\rho}^{*} \Pi(\rho)=\Pi(\rho) \kappa_{\rho}=0$ and $\tau^{*} \dot{\Pi}(\rho) \kappa=1$, then $T^{\frac{1}{2}}(\hat{\rho}-\rho)$ has the same limit distribution as

$$
T^{\frac{1}{2}} \tilde{\tau}_{\rho}^{*}(\hat{A}-A) \tilde{\kappa}_{\rho}=-T^{\frac{1}{2}} \tau_{\rho}^{*} \hat{\Pi}(\rho) \kappa_{\rho},
$$

that is, asymptotically Gaussian with mean zero.

If $\rho_{k}$ and $\rho_{l}$ are simple real roots the asymptotic covariance is

$$
\operatorname{as} \operatorname{Cov}\left(T^{\frac{1}{2}} d \hat{\rho}_{k}, T^{\frac{1}{2}} d \hat{\rho}_{l}\right)=\tau_{k}^{\prime} \Omega \tau_{l} \tilde{\kappa}_{k}^{\prime} \Sigma^{-1} \tilde{\kappa}_{l} .
$$

If $\rho_{k}$ and $\rho_{l}$ are simple complex roots the asymptotic covariances are given in terms of the matrices

$$
\begin{gathered}
\omega^{k l}=\left(\begin{array}{cc}
\omega_{r r}^{k l} & \omega_{r i}^{k l} \\
\omega_{i r}^{k l} & \omega_{i i}^{k l}
\end{array}\right)=\left(\begin{array}{cc}
\tau_{r k}^{\prime} \Omega \tau_{r l} & \tau_{r k}^{\prime} \Omega \tau_{i l} \\
\tau_{i k}^{\prime} \Omega \tau_{r l} & \tau_{i k}^{\prime} \Omega \tau_{i l}
\end{array}\right), \\
\gamma^{k l}=\left(\begin{array}{cc}
\gamma_{r r}^{k l} & \gamma_{r i}^{k l} \\
\gamma_{i r}^{k l} & \gamma_{i i}^{k l}
\end{array}\right)=\left(\begin{array}{cc}
\tilde{\kappa}_{r k}^{\prime} \Sigma^{-1} \tilde{\kappa}_{r l} & \tilde{\kappa}_{r k}^{\prime} \Sigma^{-1} \tilde{\kappa}_{i l} \\
\tilde{\kappa}_{i k}^{\prime} \Sigma^{-1} \tilde{\kappa}_{r l} & \tilde{\kappa}_{i k}^{\prime} \Sigma^{-1} \tilde{\kappa}_{i l}
\end{array}\right) .
\end{gathered}
$$

The asymptotic variances and covariances of the estimated real and imaginary part of the roots are given by

$$
\begin{aligned}
a s \operatorname{Cov}\left(T^{\frac{1}{2}} d \hat{\rho}_{r k}, T^{\frac{1}{2}} d \hat{\rho}_{r l}\right) & =\omega_{r r}^{k l} \gamma_{r r}^{k l}+\omega_{r i}^{k l} \gamma_{r i}^{k l}+\omega_{i r}^{k l} \gamma_{i r}^{k l}+\omega_{i i}^{k l} \gamma_{i i}^{k l} \\
a s \operatorname{Cov}\left(T^{\frac{1}{2}} d \hat{\rho}_{i k}, T^{\frac{1}{2}} d \hat{\rho}_{i l}\right) & =\omega_{r r}^{k l} \gamma_{i i}^{k l}-\omega_{r i}^{k l} \gamma_{i r}^{k l}-\omega_{i r}^{k l} \gamma_{r i}^{k l}+\omega_{i i}^{k l} \gamma_{r r}^{k l} \\
a s \operatorname{Cov}\left(T^{\frac{1}{2}} d \hat{\rho}_{r k}, T^{\frac{1}{2}} d \hat{\rho}_{i l}\right) & =\omega_{r r}^{k l} \gamma_{r i}^{k l}-\omega_{i i}^{k l} \gamma_{i r}^{k l}-\omega_{r i}^{k l} \gamma_{r r}^{k l}+\omega_{i r}^{k l} \gamma_{i i}^{k l}
\end{aligned}
$$


Proof. From the continuity of $\rho(\Pi)$ and the consistency of $\hat{\Pi}$, it follows that also $\hat{\rho}$ is consistent. From the differential (10) and (15), it follows that

$$
T^{\frac{1}{2}}(\hat{\rho}-\rho)=T^{\frac{1}{2}} \tilde{\tau}_{\rho}^{*}(\hat{A}-A) \tilde{\kappa}_{\rho}+o_{P}(1)=-T^{\frac{1}{2}} \tau_{\rho}^{*} \hat{\Pi}(\rho) \kappa_{\rho}+o_{P}(1)
$$

which should be compared with Wymer (1972, page 575), where $\operatorname{vec}\left(\operatorname{diag}\left(d \hat{\rho}_{1}, \ldots, d \hat{\rho}_{m}\right)\right)$ is found in terms of a matrix derived from the eigenvectors.

We first assume that $\rho_{k}$ and $\rho_{l}$ are two simple real roots, in which case we get from $(22)$

$$
\operatorname{as} \operatorname{Cov}\left(T^{\frac{1}{2}} d \hat{\rho}_{k}, T^{\frac{1}{2}} d \hat{\rho}_{l}\right)=\tilde{\tau}_{k}^{\prime} B \Omega B^{\prime} \tilde{\tau}_{l} \tilde{\kappa}_{k}^{\prime} \Sigma^{-1} \tilde{\kappa}_{l},
$$

which proves (18).

If $\rho_{k}$ and $\rho_{l}$ are two simple complex roots the formulae are somewhat more complicated. We get from (15) that

$$
\begin{aligned}
d \hat{\rho} & =\left(\tilde{\tau}_{r}^{\prime}-i \tilde{\tau}_{i}^{\prime}\right) d \hat{A}\left(\tilde{\kappa}_{r}+i \tilde{\kappa}_{i}\right) \\
& =\tilde{\tau}_{r}^{\prime}(d \hat{A}) \tilde{\kappa}_{r}+\tilde{\tau}_{i}^{\prime}(d \hat{A}) \tilde{\kappa}_{i}+i\left(\tilde{\tau}_{r}^{\prime}(d \hat{A}) \tilde{\kappa}_{i}-\tilde{\tau}_{i}^{\prime}(d \hat{A}) \tilde{\kappa}_{r}\right), \\
& =d \hat{\rho}_{r}+i d \hat{\rho}_{i},
\end{aligned}
$$

and hence in terms of $\omega^{k l}$ and $\gamma^{k l}$, we find the results in (19), (20), and (21).

If all the roots are simple the variances can be expressed in terms of the quantities $\tau_{k}^{*} \Omega \tau_{l}, \rho_{k}$, and $\rho_{l}$ :

Corollary 4 Let $X_{t}$ given by (1) be stationary and assume that all roots are simple. We define the matrix $\Theta$ with elements

$$
\Theta_{k l}=\frac{\tau_{k}^{*} \Omega \tau_{l}}{1-\rho_{k} \bar{\rho}_{l}}
$$

If all roots are real we get

$$
\operatorname{as} \operatorname{Cov}\left(T^{\frac{1}{2}} d \hat{\rho}_{k}, T^{\frac{1}{2}} d \hat{\rho}_{l}\right)=\tau_{k}^{\prime} \Omega \tau_{l}\left(\frac{\tau_{i}^{\prime} \Omega \tau_{j}}{1-\rho_{i} \rho_{j}}\right)^{k l}=\tau_{k}^{\prime} \Omega \tau_{l} \Theta^{k l} .
$$


In general, if all roots are possibly complex, we have the expressions (19), (20), and (21) with

$$
\gamma^{k l}=\frac{1}{2}\left(\begin{array}{rr}
\Theta_{r}^{k l}+\Theta_{r}^{k \bar{l}} & \Theta_{i}^{k l}-\Theta_{i}^{k \bar{l}} \\
-\Theta_{i}^{k l}-\Theta_{i}^{k \bar{l}} & \Theta_{r}^{k l}-\Theta_{r}^{k l}
\end{array}\right),
$$

where $\bar{l}$ is defined by $\rho_{\bar{l}}=\bar{\rho}_{l}$.

Proof. From (13) it follows that $\tilde{\tau}_{k}^{*} B=\tau_{k}^{*}$, and from (17) we get

$$
\tilde{\tau}_{k}^{*} \Sigma \tilde{\tau}_{l}=\tilde{\tau}_{k}^{*} A \Sigma A^{\prime} \tilde{\tau}_{l}+\tilde{\tau}_{k}^{*} B \Omega B^{\prime} \tilde{\tau}_{l}=\rho_{k} \bar{\rho}_{l} \tilde{\tau}_{k}^{*} \Sigma \tilde{\tau}_{l}+\tau_{k}^{*} \Omega \tau_{l}
$$

since $A^{\prime} \tilde{\tau}_{l}=\bar{\rho}_{l} \tilde{\tau}_{l}$, and hence

$$
\tilde{\tau}_{k}^{*} \Sigma \tilde{\tau}_{l}=\frac{\tau_{k}^{*} \Omega \tau_{l}}{1-\rho_{k} \bar{\rho}_{l}}=\Theta_{k l} .
$$

Note that $\Theta$ in general is a complex matrix which is self-adjoint: $\Theta=\Theta^{*}$. For $\tau=\left(\tilde{\tau}_{1}, \ldots, \tilde{\tau}_{m}\right)$ we get

$$
\tilde{\kappa}_{k}^{*} \Sigma^{-1} \tilde{\kappa}_{l}=\tilde{\kappa}_{k}^{*} \tau\left(\tau^{*} \Sigma \tau\right)^{-1} \tau^{*} \tilde{\kappa}_{l}=e_{k}^{\prime} \Theta^{-1} e_{l}=\Theta^{k l},
$$

the $k l^{\prime}$ th element of $\Theta^{-1}$. This proves (23).

From the relations

$$
\begin{aligned}
& \Theta^{k l}=\tilde{\kappa}_{k}^{*} \Sigma^{-1} \tilde{\kappa}_{l}=\left(\tilde{\kappa}_{r k}^{\prime}-i \tilde{\kappa}_{i k}^{\prime}\right) \Sigma^{-1}\left(\tilde{\kappa}_{r l}+i \tilde{\kappa}_{i l}\right)=\gamma_{r r}^{k l}+\gamma_{i i}^{k l}+i\left(\gamma_{r i}^{k l}-\gamma_{i r}^{k l}\right) \\
& \Theta^{k l}=\tilde{\kappa}_{k}^{*} \Sigma^{-1} \tilde{\kappa}_{l}=\left(\tilde{\kappa}_{r k}^{\prime}-i \tilde{\kappa}_{i k}^{\prime}\right) \Sigma^{-1}\left(\tilde{\kappa}_{r l}-i \tilde{\kappa}_{i l}\right)=\gamma_{r r}^{k l}-\gamma_{i i}^{k l}-i\left(\gamma_{r i}^{k l}+\gamma_{i r}^{k l}\right)
\end{aligned}
$$

we find the expression (24) for $\gamma^{k l}$.

A simple example is a bivariate system with one lag and two real roots, where the asymptotic variance of the estimated roots is

$$
\left(\begin{array}{cc}
\tau_{1}^{\prime} \Omega \tau_{1} & \tau_{1}^{\prime} \Omega \tau_{2} \\
\tau_{2}^{\prime} \Omega \tau_{1} & \tau_{2}^{\prime} \Omega \tau_{2}
\end{array}\right) \bullet\left(\begin{array}{cc}
\frac{\tau_{1}^{\prime} \Omega \tau_{1}}{1-\rho_{1}^{2}} & \frac{\tau_{1}^{\prime} \Omega \tau_{2}}{1-\rho_{1} \rho_{2}} \\
\frac{\tau_{2} \Omega \tau_{1}}{1-\rho_{1} \rho 2} & \frac{\tau_{2}^{\prime} \Omega \tau_{2}}{1-\rho_{2}^{2}}
\end{array}\right)^{-1} .
$$

Here the $\bullet$ denotes the element-wise product. For a univariate system with one lag this simplifies to the well-known result that the asymptotic variance is $1-\rho^{2}$. It is seen from (26) and in general (18) that 
the expression for the asymptotic variance matrix is independent of the normalization of the eigenvectors $\left\{\tau_{k}\right\}$.

The matrix $\Theta^{-1}$ can be calculated using real matrices as follows. The complex number $\tau_{k}^{*} \Omega \tau_{l}$ is represented by the matrix

$$
\left(\begin{array}{cc}
\tau_{r k}^{\prime} & \tau_{i k}^{\prime} \\
-\tau_{i k}^{\prime} & \tau_{r k}^{\prime}
\end{array}\right)\left(\begin{array}{cc}
\Omega & 0 \\
0 & \Omega
\end{array}\right)\left(\begin{array}{cc}
\tau_{r l} & -\tau_{i l} \\
\tau_{i l} & \tau_{r l}
\end{array}\right)
$$

and $\left(1-\rho_{k} \bar{\rho}_{l}\right)$ by

$$
\left(\begin{array}{ll}
1 & 0 \\
0 & 1
\end{array}\right)-\left(\begin{array}{rr}
\rho_{r k} & -\rho_{i k} \\
\rho_{i k} & \rho_{r k}
\end{array}\right)\left(\begin{array}{rr}
\rho_{r l} & \rho_{i l} \\
-\rho_{i l} & \rho_{r l}
\end{array}\right)
$$

In this way we obtain an expression for $\Theta$ as a $2 m \times 2 m$ matrix, the inverse of which represents $\Theta^{-1}$ with $2 \times 2$ blocks of the form

$$
\Theta^{k l}=\left(\begin{array}{cc}
\Theta_{r}^{k l} & -\Theta_{i}^{k l} \\
\Theta_{i}^{k l} & \Theta_{r}^{k l}
\end{array}\right)
$$

\section{The cointegrated VAR}

In this section we show how the above results of Section 3 hold for the stationary roots in a cointegrated $I(1)$ process. Let $X_{t}$ be an $I(1)$ process given by the vector autoregressive model (1), but reparametrized as

$$
\Delta X_{t}=\alpha \beta^{\prime} X_{t-1}+\Gamma_{1} \Delta X_{t-1}+\Gamma_{2} \Delta X_{t-2}+\varepsilon_{t} .
$$

The parameters are related by

$$
\begin{array}{ll}
\Pi_{1}=I_{n}+\alpha \beta^{\prime}+\Gamma_{1}, & \alpha \beta^{\prime}=\Pi_{1}+\Pi_{2}+\Pi_{3}-I_{n}, \\
\Pi_{2}=-\Gamma_{1}+\Gamma_{2}, & \Gamma_{1}=-\Pi_{2}-\Pi_{3}, \\
\Pi_{3}=-\Gamma_{2}, & \Gamma_{2}=-\Pi_{3} .
\end{array}
$$

In terms of the new parameters we find

$$
\Pi(\rho)=-\rho^{2}(1-\rho) I_{n}-\rho^{2} \alpha \beta^{\prime}+\rho(1-\rho) \Gamma_{1}+(1-\rho) \Gamma_{2} .
$$


Note that $\Pi(1)=-\alpha \beta^{\prime}$, has reduced rank, which causes the nonstationarity of $X_{t}$. From the companion form (11) we derive the companion form for $Y_{t}=\left(X_{t}^{\prime} \beta_{\perp}, X_{t}^{\prime} \beta, \Delta X_{t}^{\prime}, \Delta X_{t-1}^{\prime}\right)^{\prime}$

$$
Y_{t}=K A K^{-1} Y_{t-1}+K B \varepsilon_{t}=M Y_{t-1}+N \varepsilon_{t},
$$

with

$$
K=\left(\begin{array}{ccc}
\beta_{\perp}^{\prime} & 0 & 0 \\
\beta^{\prime} & 0 & 0 \\
I_{n} & -I_{n} & 0 \\
0 & I_{n} & -I_{n}
\end{array}\right), K^{-1}=\left(\begin{array}{cccc}
\bar{\beta}_{\perp} & \bar{\beta} & 0 & 0 \\
\bar{\beta}_{\perp} & \bar{\beta} & -I_{n} & 0 \\
\bar{\beta}_{\perp} & \bar{\beta} & -I_{n} & -I_{n}
\end{array}\right)
$$

and

$$
M=K A K^{-1}=\left(\begin{array}{cccc}
I_{n-r} & \beta_{\perp}^{\prime} \alpha & \beta_{\perp}^{\prime} \Gamma_{1} & \beta_{\perp}^{\prime} \Gamma_{2} \\
0 & I_{r}+\beta^{\prime} \alpha & \beta^{\prime} \Gamma_{1} & \beta^{\prime} \Gamma_{2} \\
0 & \alpha & \Gamma_{1} & \Gamma_{2} \\
0 & 0 & I_{n} & 0
\end{array}\right), N=K B=\left(\begin{array}{c}
\beta_{\perp}^{\prime} \\
\beta^{\prime} \\
I_{n} \\
0
\end{array}\right) .
$$

For the stationary process $Z_{t}^{\prime}=\left(X_{t}^{\prime} \beta, \Delta X_{t}^{\prime}, \Delta X_{t-1}^{\prime}\right)^{\prime}$ we have the equations

$$
Z_{t}=P Z_{t-1}+Q \varepsilon_{t}
$$

with

$$
P=\left(\begin{array}{ccc}
I_{r}+\beta^{\prime} \alpha & \beta^{\prime} \Gamma_{1} & \beta^{\prime} \Gamma_{2} \\
\alpha & \Gamma_{1} & \Gamma_{2} \\
0 & I_{n} & 0
\end{array}\right), Q=\left(\begin{array}{c}
\beta^{\prime} \\
I_{n} \\
0
\end{array}\right) .
$$

It follows from (12) and (13) that the eigenvectors of $M$ satisfy

$$
\begin{aligned}
& \kappa_{\rho}^{\prime}(M)=\kappa_{\rho}^{\prime}\left(\rho^{2} I_{n}, \rho I_{n}, I_{n}\right) K^{\prime}, \\
& \tau_{\rho}^{*}(M)=\tau_{\rho}^{*}\left(I_{n}, \rho I_{n}-\Pi_{1}, \rho^{2} I_{n}-\rho \Pi_{1}-\Pi_{2}\right) K^{-1} .
\end{aligned}
$$

A calculation then shows the following result:

Lemma 5 If $X_{t}$ is an I(1) process given by (27), then the companion matrix $P$ for the stationary part of the process and the companion matrix 
$M$ have the same eigenvalues apart from the unit roots of $M$. If $\rho$ is a root of $M$, then a right eigenvector is given by

$$
\kappa_{\rho}^{\prime}(M)=\kappa_{\rho}^{\prime}\left(\rho^{2} \beta_{\perp}, \rho^{2} \beta,(\rho-1) \rho I_{n},(\rho-1) I_{n}\right),
$$

where $\Pi(\rho) \kappa_{\rho}=0$, and a left eigenvector is

$$
\tau_{\rho}^{*}(M)=\tau_{\rho}^{*}\left(0, \frac{1}{\rho-1} \alpha, \rho^{-2}\left(\rho \Gamma_{1}+\Gamma_{2}\right), \rho^{-1} \Gamma_{2}\right),
$$

where $\tau_{\rho}^{*} \Pi(\rho)=0$. The corresponding eigenvectors of $P$, for $\rho \neq 1$, are

$$
\begin{aligned}
& \breve{\kappa}_{\rho}^{\prime}=\kappa_{\rho}^{\prime}\left(\rho^{2} \beta,(\rho-1) \rho I_{n},(\rho-1) I_{n}\right), \\
& \breve{\tau}_{\rho}^{*}=\tau_{\rho}^{*}\left(\frac{1}{\rho-1} \alpha, \rho^{-2}\left(\rho \Gamma_{1}+\Gamma_{2}\right), \rho^{-1} \Gamma_{2}\right),
\end{aligned}
$$

which are normalized on

$$
\breve{\tau}_{\rho}^{*} \breve{\kappa}_{\rho}=\tau_{\rho}^{*} \dot{\Pi}(\rho) \kappa_{\rho}=1 .
$$

\subsection{Asymptotic distribution of eigenvalues and im- proved approximation for $\rho$ close to 1}

In a cointegrated VAR we estimate the coefficients $\alpha$ and $\beta$ by reduced rank regression of $\Delta X_{t}$ on $X_{t-1}$ corrected for $\Delta X_{t-1}, \Delta X_{t-2}$, see Johansen (1996) or Anderson (1951). Because the estimate of $\beta$ is $T^{-1}$ consistent, we determine the asymptotic distribution of the other coefficients by regression of $\Delta X_{t}$ on $\left(\beta^{\prime} X_{t-1}, \Delta X_{t-1}, \Delta X_{t-2}\right)$ where $\beta$ is replaced by $\hat{\beta}$. We find that $T^{\frac{1}{2}}\left(\hat{\alpha}-\alpha, \hat{\Gamma}_{1}-\Gamma_{1}, \hat{\Gamma}_{2}-\Gamma_{2}\right)$ is asymptotically Gaussian with mean zero and variance

$$
\Omega \otimes \Psi^{-1},
$$

where $\Psi$ denotes the variance of the stationary part of the stacked process

$$
\Psi=\operatorname{Var}\left(\beta^{\prime} X_{t}, \Delta X_{t}, \Delta X_{t-1}\right),
$$

which, as in the stationary case, can be found from the equations

$$
\Psi=P \Psi P^{\prime}+Q \Omega Q^{\prime} .
$$


From

$$
T^{\frac{1}{2}}(\hat{P}-P)=T^{\frac{1}{2}} Q\left(\hat{\alpha}-\alpha, \hat{\Gamma}_{1}-\Gamma_{1}, \hat{\Gamma}_{2}-\Gamma_{2}\right)
$$

we find

$$
T^{\frac{1}{2}} \breve{\tau}_{\rho}^{*}(\hat{P}-P) \breve{\kappa}_{\rho}=-T^{\frac{1}{2}} \breve{\tau}_{\rho}^{*} Q(\hat{\Pi}(\rho)-\Pi(\rho)) \kappa_{\rho}=-T^{\frac{1}{2}} \tau_{\rho}^{*}(\hat{\Pi}(\rho)-\Pi(\rho)) \kappa_{\rho} .
$$

As in (15) we find

$$
d \hat{\rho}=\breve{\tau}_{\rho}^{*}(d \hat{P}) \breve{\kappa}_{\rho},
$$

and we can summarize the results in

Theorem 6 Let $X_{t}$ be the cointegrated I(1) process given by (27), and let $|\Pi(\rho)|=0$ have $n-r$ unit roots and define

$$
\Psi=\operatorname{Var}\left(\beta^{\prime} X_{t}, \Delta X_{t}, \Delta X_{t-1}\right)
$$

then the conclusions of Theorem 3 and Corollary 4 hold with $\Sigma$ replaced by $\Psi$.

The above result holds for stationary roots. In order to see what happens if a root $\rho_{1}$, say, tends to 1 , consider the result for two real roots, see (26). In this case the variance is

$$
\left(\begin{array}{cc}
\left(1-\rho_{1}^{2}\right) \tau_{1}^{\prime} \Omega \tau_{1} & \mu \tau_{1}^{\prime} \Omega \tau_{2} \\
\mu \tau_{2}^{\prime} \Omega \tau_{1} & \left(1-\rho_{2}^{2}\right) \tau_{2}^{\prime} \Omega \tau_{2}
\end{array}\right) \bullet\left(\begin{array}{cc}
\tau_{1}^{\prime} \Omega \tau_{1} & \nu \tau_{1}^{\prime} \Omega \tau_{2} \\
\nu \tau_{2}^{\prime} \Omega \tau_{1} & \tau_{2}^{\prime} \Omega \tau_{2}
\end{array}\right)^{-1}
$$

with

$$
\mu=\sqrt{\left(1-\rho_{1}^{2}\right)\left(1-\rho_{2}^{2}\right)}, \nu=\frac{\sqrt{\left(1-\rho_{1}^{2}\right)\left(1-\rho_{2}^{2}\right)}}{1-\rho_{1} \rho_{2}} .
$$

For $\rho_{1} \rightarrow 1$ it holds that $\mu \rightarrow 0$ and $\nu \rightarrow 0$, and the variance matrix converges towards

$$
\left(\begin{array}{cc}
0 & 0 \\
0 & \left(1-\rho_{2}^{2}\right) \tau_{2}^{\prime} \Omega \tau_{2}
\end{array}\right) \bullet\left(\begin{array}{cc}
\tau_{1}^{\prime} \Omega \tau_{1} & 0 \\
0 & \tau_{2}^{\prime} \Omega \tau_{2}
\end{array}\right)^{-1}=\left(\begin{array}{cc}
0 & 0 \\
0 & \left(1-\rho_{2}^{2}\right)
\end{array}\right)
$$


Thus the variance of $\hat{\rho}_{1}$ tends to zero for $\rho_{1} \rightarrow 1$, and simulations show that we get a very skew and concentrated distribution. It is therefore convenient to transform the roots before confidence interval are found.

For $y=\sin x, x \in]-\frac{1}{2} \pi, \frac{1}{2} \pi[$, we find $x=\operatorname{Arcsin}(y), y \in]-1,1[$ and $d x / d y=1 / \sqrt{\left(1-x^{2}\right)}$. We introduce the Arcsin transformation of the roots which eliminates the factor $\left(1-\rho^{2}\right)$ and find

Theorem 7 If all roots are real and distinct the asymptotic distribution of

$$
T^{\frac{1}{2}}\left(\operatorname{Arcsin}\left(\hat{\rho}_{k}\right)-\operatorname{Arcsin}\left(\rho_{k}\right)\right), k=1, \ldots, m
$$

is Gaussian with mean zero and variance matrix given by

$$
\left\{\tau_{l}^{\prime} \Omega \tau_{k}\right\} \bullet\left\{\tau_{l}^{\prime} \Omega \tau_{k} \nu_{k l}\right\}^{-1}
$$

with $\nu_{k l}$ given by

$$
\nu_{k l}=\frac{\sqrt{\left(1-\rho_{k}^{2}\right)\left(1-\rho_{l}^{2}\right)}}{1-\rho_{k} \rho_{l}} .
$$

Proof. The asymptotic distribution of $\left\{T^{\frac{1}{2}}\left(\hat{\rho}_{k}-\rho_{k}\right)\right\}$ has covariance matrix with elements given by (23). It follows that the asymptotic covariance matrix of the vector

$$
\left\{T^{\frac{1}{2}}\left(\operatorname{Arcsin}\left(\hat{\rho}_{k}\right)-\operatorname{Arcsin}\left(\rho_{k}\right)\right)\right\}
$$

is given by the matrix with elements

$$
\frac{\tau_{k}^{\prime} \Omega \tau_{l}}{\sqrt{\left(1-\rho_{k}^{2}\right)\left(1-\rho_{l}^{2}\right)}}\left(\frac{\tau_{i}^{\prime} \Omega \tau_{j}}{1-\rho_{i} \rho_{j}}\right)^{k l}=\tau_{k}^{\prime} \Omega \tau_{l}\left(\sqrt{\left(1-\rho_{i}^{2}\right)\left(1-\rho_{j}^{2}\right)} \frac{\tau_{i}^{\prime} \Omega \tau_{j}}{1-\rho_{i} \rho_{j}}\right)^{k l} .
$$




\section{Simulations}

In this section we simulate a bivariate system in order to get an impression of the distributions and some confidence intervals. We take $T=100$ and the number of simulation is 200 . We simulate the system

$$
\left(\begin{array}{c}
X_{1 t} \\
X_{2 t}
\end{array}\right)=\left(\begin{array}{ll}
A_{11} & A_{12} \\
A_{21} & A_{22}
\end{array}\right)\left(\begin{array}{c}
X_{1 t-1} \\
X_{2 t-1}
\end{array}\right)+\left(\begin{array}{l}
\varepsilon_{1 t} \\
\varepsilon_{2 t}
\end{array}\right)
$$

where $\varepsilon_{t}$ are i.i.d. $N(0, \Omega)$, with

$$
\Omega=\left(\begin{array}{ll}
1.25 & 1.55 \\
1.55 & 2.98
\end{array}\right)
$$

and $A$ is chosen to give suitable roots. Figure 1 shows the estimated roots if we have a process with the roots $\pm 0.5 i$. It is seen that the real and imaginary part have a joint distribution which is described by the variance matrix given by the relations (19), (20), and (21).

Next consider in Figure 2 the simulation of a system with two real roots at \pm 0.5 . It is seen that all estimated roots are real, and the distribution is given by the variance in (18). If, however, we consider Figure 3 , where the roots are 0.9 and 0.5 , we see that the empirical distribution spreads into the complex plane, a phenomenon that is not captured by the asymptotic distribution, which is concentrated on the real axis. Thus the finite sample performance of the estimated real roots needs further study.

An explanation of this phenomenon is the following. The characteristic equation is of second degree and the roots are found as the intersection of a parabola with the $x$ axis. If the estimated parabola is sufficiently close to the true one, assumed to have two real roots, the estimated roots are real too. If, however, the random coefficients have perturbed the parabola so much that it does not intersect the $x$ axis, then the roots become complex.

This is a relevant observation also for a discussion of unit roots, where one sometimes finds that there are two complex roots with rather 
large absolute value. The question then is whether one of them or possibly both are unit roots. Obviously if we have two unit roots we sometimes will observe two complex roots with a high modulus, but even if we have roots at 1.0 and 0.9 , say, the random variation could be such that we observe two large complex roots with a real part of .95.

In the simulation in Figure 4 we have a root at 0.95 and a root at 0.5. It is seen in Figure 5 that the distribution is highly skewed, and the Arcsin transformation makes it a lot more symmetric in Figure 6. The last Figure shows a 95\% confidence ellipse for a bivariate time series with $T=50$ observations. The simulations were performed in RATS 4.20.

\section{$5.1 \quad$ References}

Anderson, T.W. (1951) Estimating linear restrictions on regression coefficients for multivariate normal distributions. Annals of Mathematical Statistics, 22, 327-351.

Anderson, T.W. (1971) Statistical Analysis of Time Series New York: John Wiley.

Johansen, S. (1996) Likelihood-Based Inference in Cointegrated Vector Autoregressive Models. Oxford: Oxford University Press.

Johansen, S., and Schaumburg, E. (1998) Likelihood analysis of seasonal cointegration, Journal of Econometrics 88, 301-339.

Magnus, J.R., and Neudecker, H. (1995) Matrix Differential Calculus with Applications in Statistics and Econometrics. New York: John Wiley.

WyMER, C.R. (1972) Econometric estimation of stochastic differential equation systems. Econometrica 40, 565-577. 


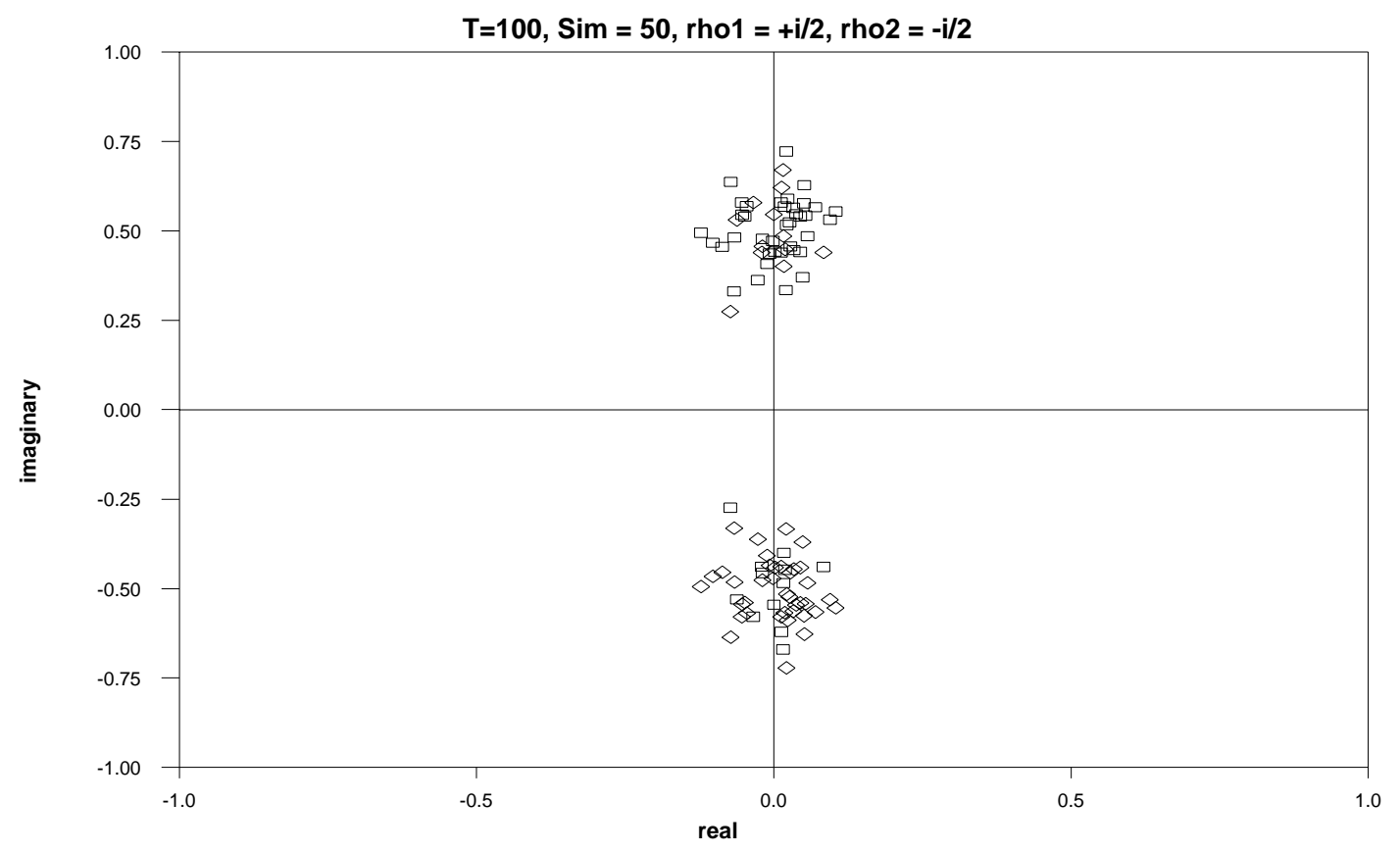

Figure 1: 


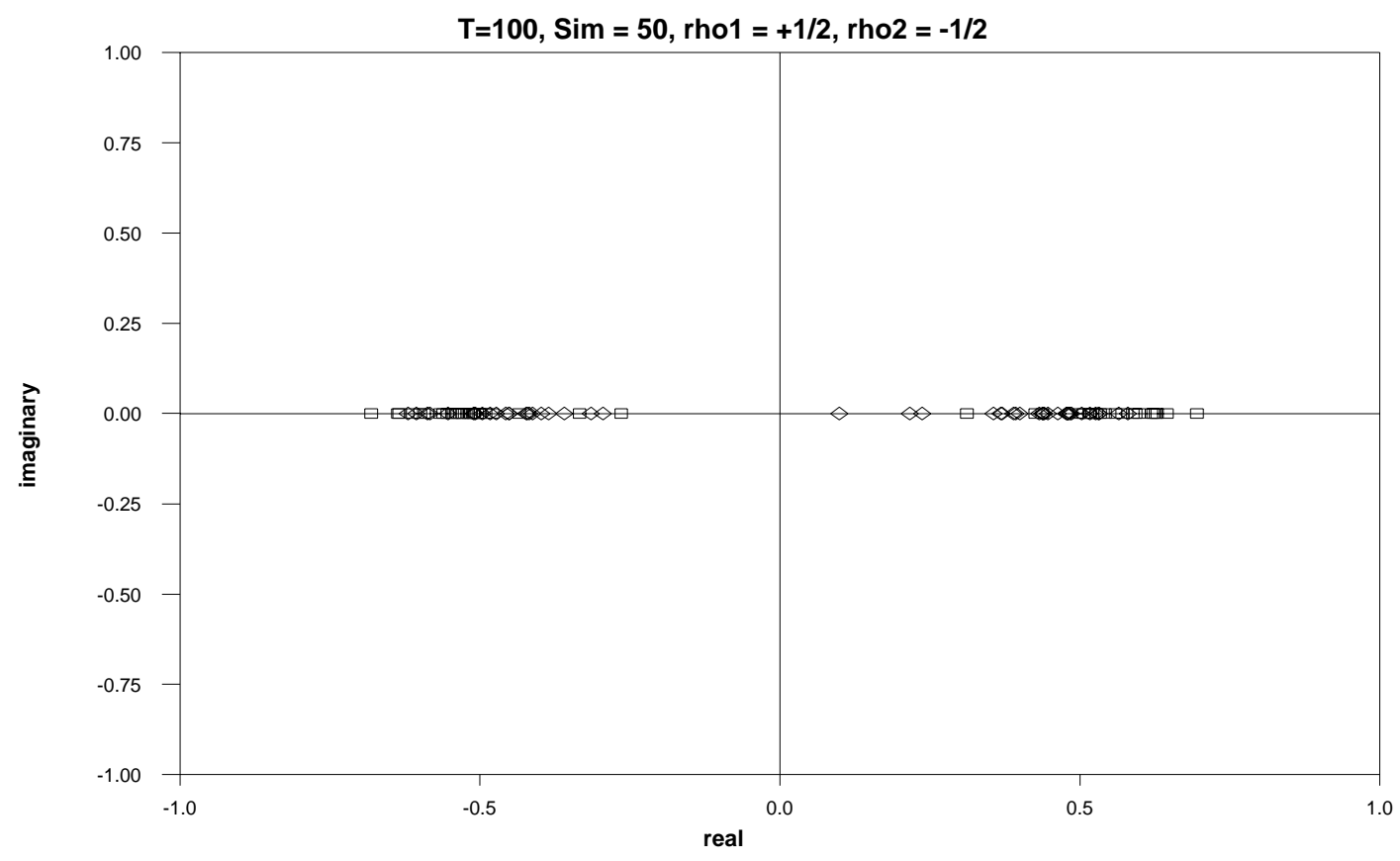

Figure 2: 


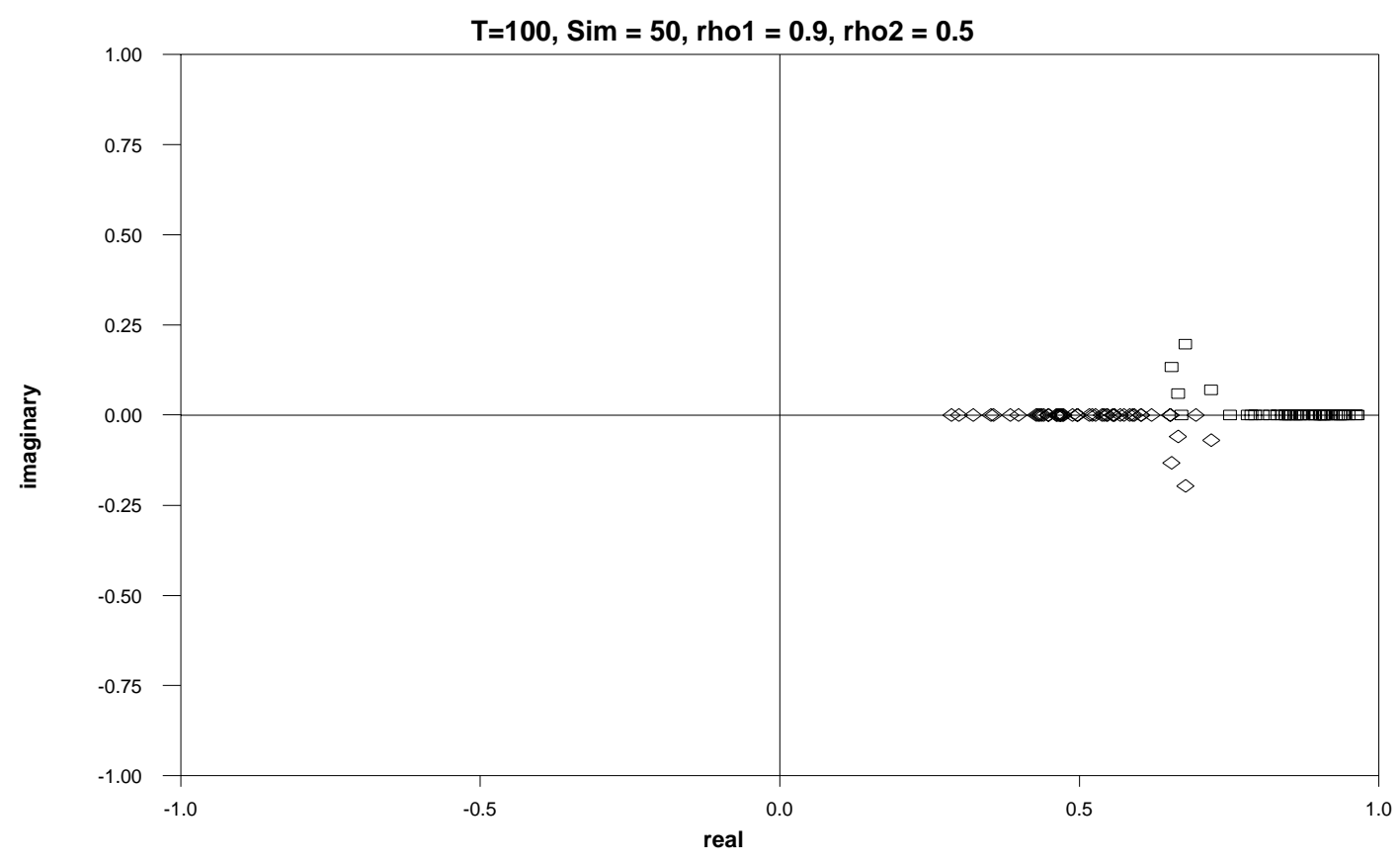

Figure 3: 


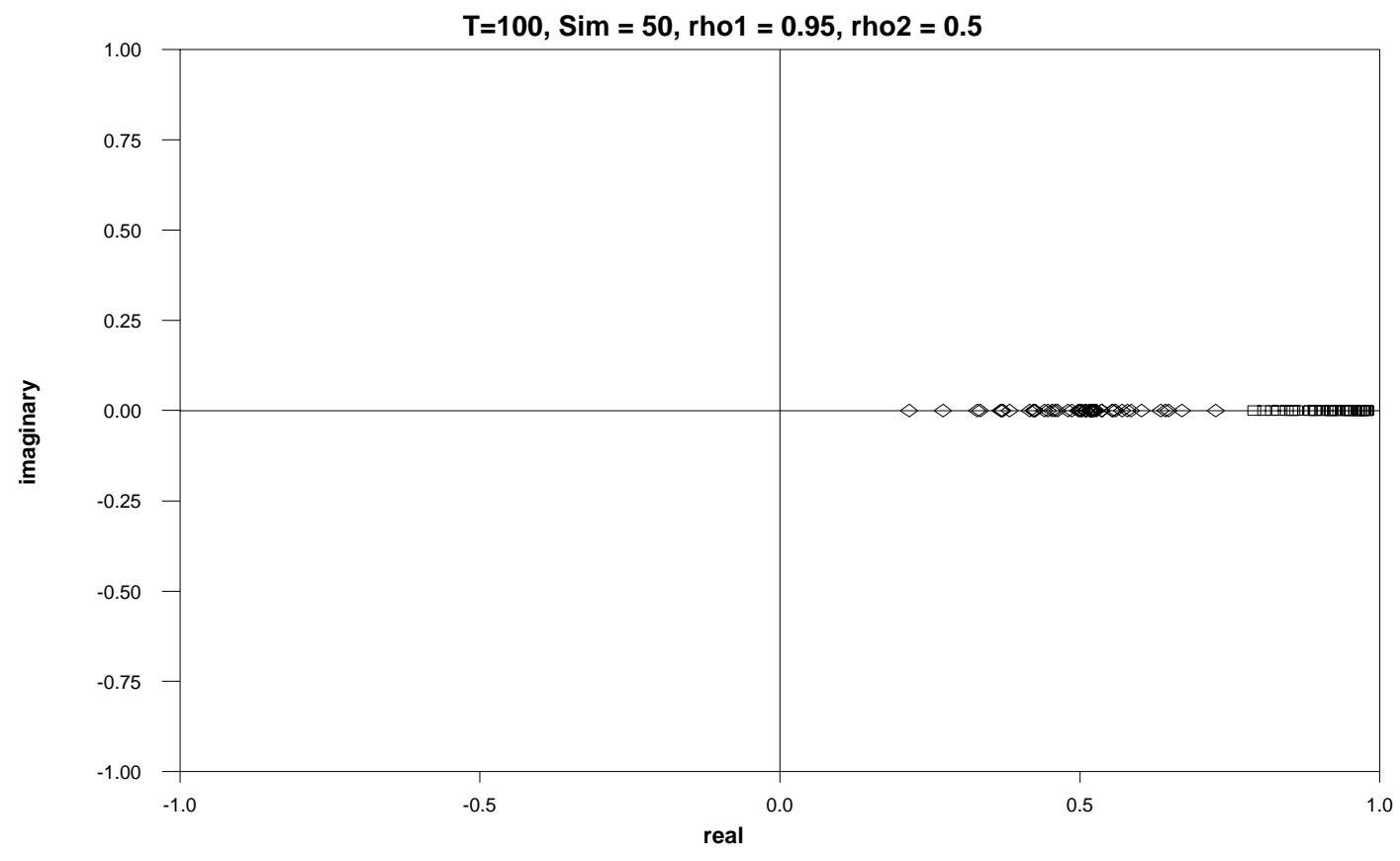

Figure 4: 


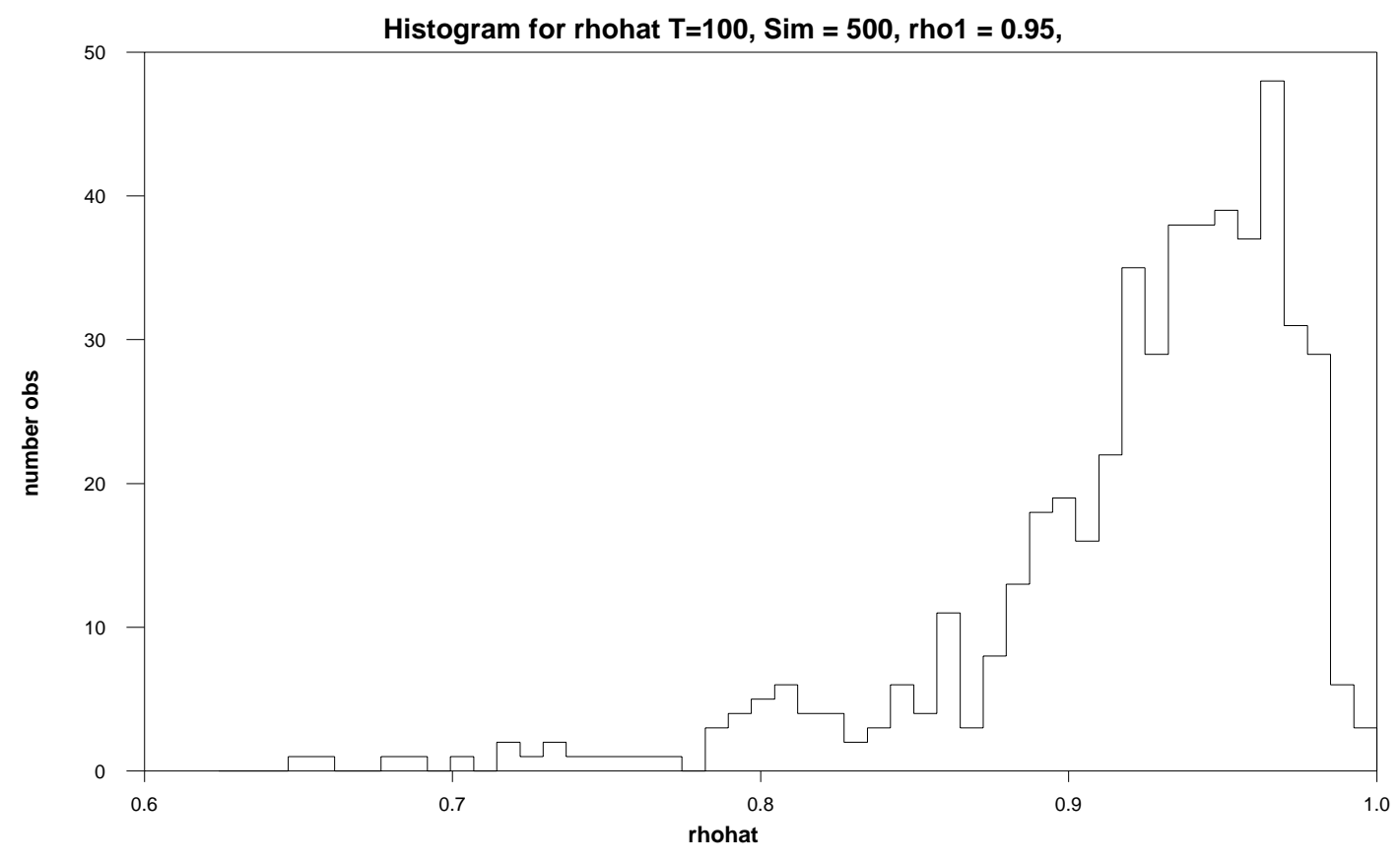

Figure 5: 


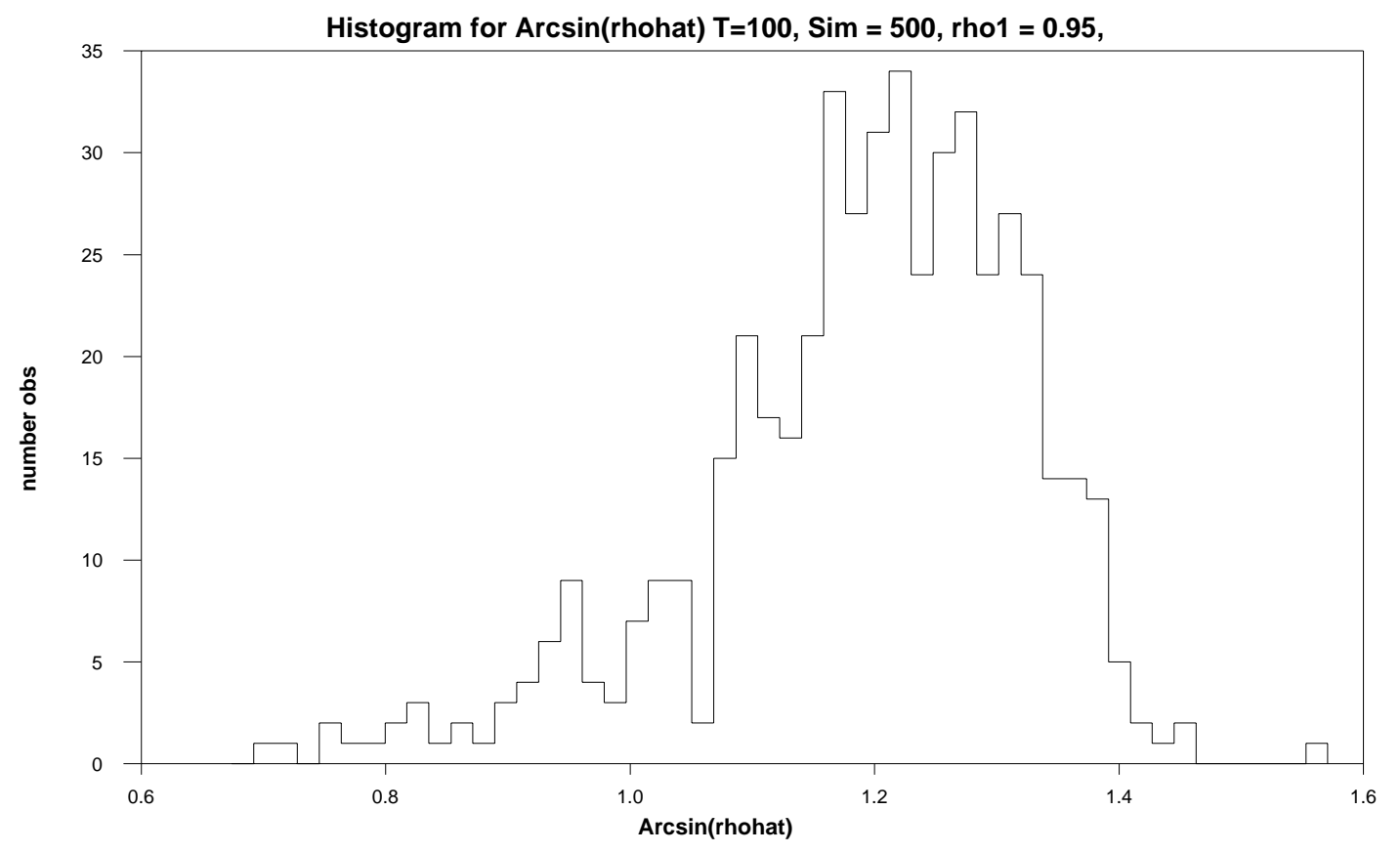

Figure 6: 


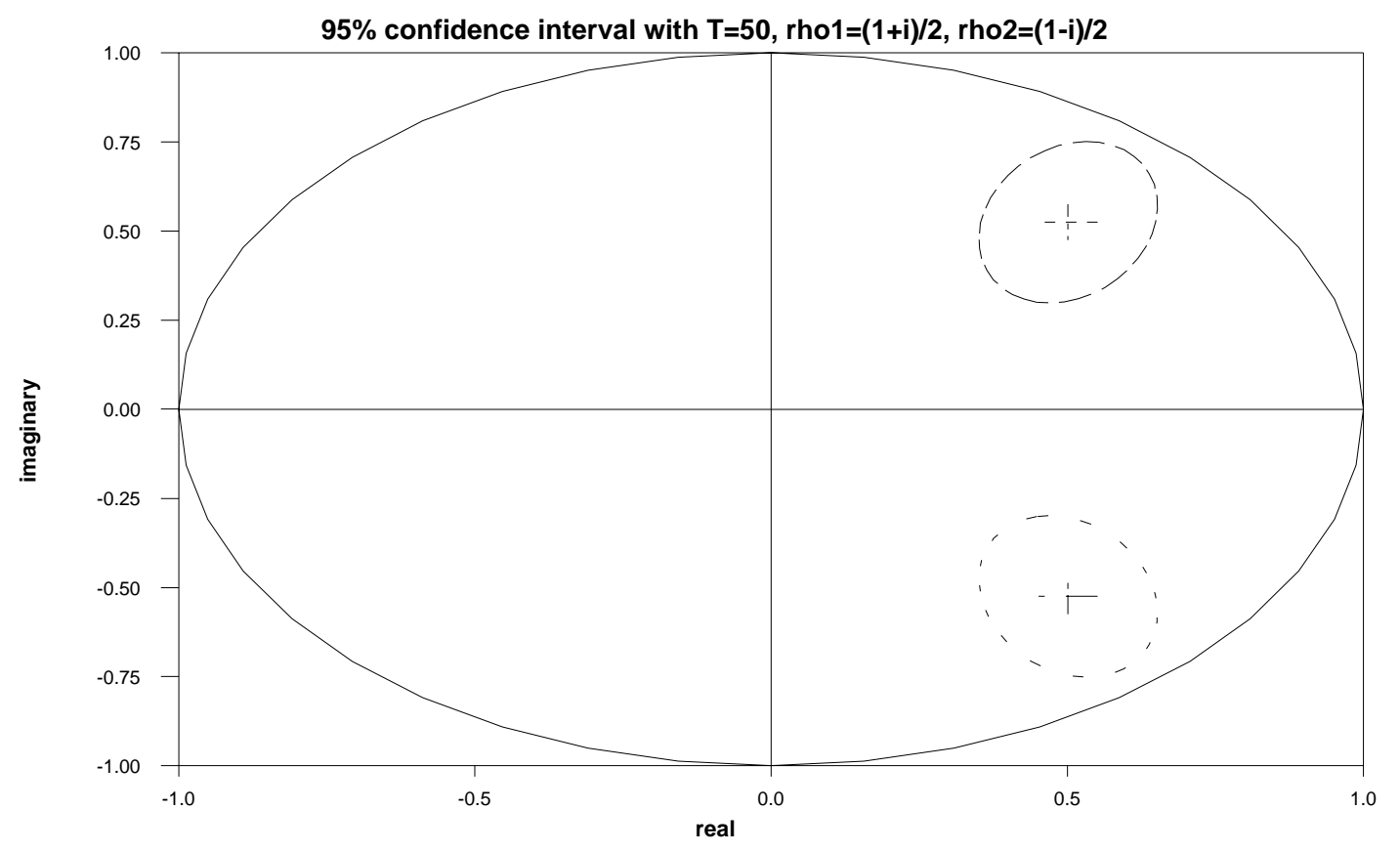

Figure 7: 